\title{
INDICADORES DE CONDICIÓN FÍSICA RELACIONADA CON LA SALUD EN UNIVERSITARIOS QUE INICIAN Y TERMINAN SUS CARRERAS
}

\author{
HEALTH-RELATED FITNESS INDICATORS AMONG UNIVERSITY \\ STUDENTS WHO BEGIN AND END THEIR CAREERS
}

Santiago Adolfo Arboleda Franco ${ }^{1}$

\section{Resumen}

El presente estudio busca determinar y comparar algunos parámetros de la condición física relacionada a la salud (CFRS) en universitarios que inician (IC) y finalizan sus carreras (FC). Se hizo un estudio descriptivo de corte transversal con 280 jóvenes de ambos sexos. Se registró información sociodemográfica, estado de salud, talla, peso, índice de masa corporal (IMC), adiposidad, flexibilidad isquiosural, potencia de miembros inferiores y aptitud cardiorrespiratoria ( $\mathrm{VO}_{2}$ máx.). Se realizó estadística descriptiva, Prueba T para muestras independientes y prueba de Levene con una significancia $\mathrm{p}<0,05$. La edad fue mayor para ambos sexos en el grupo FC. El 90,8\% de la muestra total se autorreportó sana y el 38,3\% refirió antecedentes familiares de ECNT en el primer grado de consanguinidad, principalmente hipertensión. Al comparar los grupos IC y FC divididos por sexo, hubo diferencias $(p<0,05)$ para el peso, el IMC, la adiposidad, el salto Abalakov y la $\mathrm{VO}_{2}$ máx. El CMJ solo difirió en los varones. En todos los casos las diferencias fueron desfavorables para el grupo FC. No obstante, en toda la muestra el $\mathrm{vo}_{2}$ máx. comparado con estudios de referencia fue aceptable. Se concluye que el grupo FC tuvo menores valores de CFRS, quizás atribuibles al mayor promedio de edad. El $\mathrm{VO}_{2}$ máx. estimado contradice la tendencia general sobre CFRS en universitarios.

Palabras claves: aptitud física, universitarios, riesgo de enfermedad, prevención, hábitos saludables.

\section{Abstract}

\begin{abstract}
This study aims to determine and compare some fitness health-related (FHR) parameters in university students who begin (BC) and finish their careers (FC). A cross-section study was conducted in 280 young people, both male and female. Sociodemographic data, information about the state of health, size, weight, body mass index (BMI), fattiness, hamstring flexibility, lower limb power, and cardiorespiratory fitness $\left(\mathrm{VO}_{2} \mathrm{max}\right)$ were registered. Descriptive statistics, $\mathrm{T}$ test for independent samples and Levene test with a significance level of $\mathrm{p}<0.05$ were performed. For both sexes in the FC group, individuals were older. Out of total sample, $90.8 \%$ reported themselves as healthy, and $38.3 \%$ referred a history of chronicle disease -mainly hypertension- relatives in first degree of consanguinity. When comparing both $\mathrm{BC}$ and FC groups divided by sex, there were differences $(\mathrm{p}<0.05)$ for weight, BMI, fattiness, Abalakov Jump and vo ${ }_{2}$ max. CMJ only differed on male people. For all cases, the differences were unfavourable for the FC group. Nevertheless, the $\mathrm{VO}_{2}$ max for the whole sample, compared with reference studies, was acceptable. It was concluded that the FC group had minor FHR values. This result can be associated to a higher age average. The $\mathrm{vo}_{2}$ max estimated contradicts the general FHR tendency for university students.
\end{abstract}

Keywords: fitness, university students, risk of illness, prevention, healthy behaviors.

Fecha de recepción: 15 de julio de 2014

Fecha de aprobación: 8 de noviembre de 2014

1 Doctor en Ciencias de la Actividad Física y el Deporte, Universidad de León, España. Magíster en Educación con énfasis en Fisiología del Deporte, Universidad del Valle, Colombia. Licenciado en Educación Física y Salud de la Universidad del Valle. Profesor Asistente del Área Educación Física y Deporte de la Universidad del Valle. Grupo de Investigación Incide. Correo electrónico: santiago.arboleda@correounivalle.edu.co 
Para citar este artículo:

Arboleda, S.A. (2014). Indicadores de condición física

relacionada con la salud en universitarios que inician y terminan

sus carreras. Revista Lúdica Pedagógica, (20), 93-102.

\section{INTRODUCCIÓN}

La condición física relacionada con la salud (CFRS) se entiende como un atributo ligado a la vitalidad y el desempeño físico óptimo en las actividades cotidianas, así como al disfrute de actividades de ocio y a la capacidad de responder físicamente frente a sucesos inesperados (Pagac, 2008; Garber et ál., 2011). Actualmente las recomendaciones sobre ejercicio saludable, además de centrarse en actividades moderadas que aumenten el gasto energético, reconocen la condición física como componente fundamental para preservar la salud y prevenir enfermedades (Garber et ál., 2011). No obstante, algunos estudios con adolescentes europeos han reportado una tendencia secular en la disminución de la CFRS (Moliner-Urdiales et ál., 2010a) y el descenso en los niveles saludables de acondicionamiento cardiorrespiratorio (Ortega et ál., 2011b).

La evaluación de parámetros de CFRS ha sido una estrategia de vigilancia epidemiológica para la detección temprana de riesgo frente a enfermedades crónicas no transmisibles - ECNT (Pagac, 2008). En Europa y España se ha hecho este tipo de estudios en adolescentes, como el "Estudio de Cuenca" (Vizcaíno et ál., 2002), el "Estudio Afinos" (Martínez-Gómez et ál., 2010), el "Estudio Edufit" (Ardoy et ál., 2010), el "Estudio Avena” (García, et ál., 2007), el "Estudio Helena" (Martínez-Gómez et ál., 2011) y "The European Youth Heart Study" (Ortega, Ruiz, Hurtig-Wennlöf y Sjöström, 2008). En los mismos se destaca el uso de métodos más objetivos de medición del gasto energético y la condición física, por medio de acelerometría y pruebas de aptitud física. Tales estudios han establecido diferencias entre el significado que tienen la actividad física y la condición física como predictores del riesgo cardiovascular en adolescentes. En este caso, el segundo componente tiene una mejor valoración que el simple gasto energético atribuido a la actividad física diaria. Inclusive, Ortega et ál. (2008) concluyen que la capacidad cardiovascular por sí sola está más determinada por la condición física que por la maduración sexual y la adiposidad.

No obstante, el sedentarismo como factor de riesgo y objeto de intervención de las estrategias macro de promoción y prevención es más estudiado mediante tamizajes a partir de encuestas, por la facilidad metodológica y el bajo coste (Pagac, 2008); es menos frecuente observar caracterizaciones que incluyan evaluaciones funcionales y motoras que den cuenta de la valoración directa de la aptitud física y su relación con indicadores saludables. En estudios con universitarios la tendencia metodológica es la misma, por lo que las asociaciones resultantes basadas en el autorreporte de actividades podrían arrojar imprecisiones a la hora, no solo de valorar el riesgo, sino frente a la potencialidad de las intervenciones, sobre todo las que vinculen el deporte y la actividad física al estilo de vida de los estudiantes.

Varios estudios se han aproximado a describir el perfil de riesgo de ECNT en universitarios, pero lo han hecho sobre variables muy concretas y de manera aislada. Pocos trabajos con esta población han vinculado la aptitud física relacionada con la salud en las descripciones de dicho perfil (Mendes et ál., 2006; Ramos et ál., 2009) y lo más extendido son tamizajes con métodos de observación epidemiológica que hacen aproximaciones, basadas más en el autorreporte de actividades y el gasto energético (Astudillo y Rojas, 2006; Castillo y Jiménez, 2011; Martínez-Lemos, 2009; Martins, De Castro, De Santana y Oliveira, 2008; Oviedo et ál., 2008) que en una exploración directa de las posibilidades motoras de los sujetos y su relación con los factores de riesgo. Otro aspecto a tener en cuenta es que faltan datos longitudinales que describan la dinámica de los factores de riesgo y de la CFRS en los universitarios. En Colombia pocos estudios han documentado sobre la CFRS en esta población según el tiempo transcurrido en su formación, por lo que el objetivo de esta investigación fue determinar y comparar algunos parámetros de CFRS en universitarios que inician (IC) y finalizan sus carreras (FC).

\section{MÉTODOS}

\section{Sujetos}

Participaron estudiantes de la Universidad del Valle, Cali (Colombia), aparentemente sanos que cursaban la asignatura Deporte Formativo. Para el estudio fueron clasificados en dos grupos: el primero conformado por quienes inciaban sus carreras (IC) y el segundo por quienes las finalizaban (FC). El grupo IC fue seleccionado mediante un muestreo aleatorio con fijación proporcional según el sexo, de una población total de 1194 participantes de la asignatura, resultando una muestra de 208 casos. El grupo FC se obtuvo por medio de un censo sobre los 83 sujetos que cumplieron este criterio de inclusión, con una tasa de respuesta del $84,3 \%$ totalizando 72 casos. Con todo, el total de sujetos fue de $280 \mathrm{y}$ sus principales características se exponen en la tabla 1 . 
Tabla 1. Características del grupo estudiado

\begin{tabular}{|c|c|c|c|c|}
\hline & \multicolumn{2}{|c|}{ Inicio carrera (ic) } & \multicolumn{2}{c|}{ Fin carrera (fc) } \\
\hline & $\begin{array}{c}\text { Hombres } \\
(n=97)\end{array}$ & $\begin{array}{c}\text { Mujeres } \\
(n=111)\end{array}$ & $\begin{array}{c}\text { Hombres } \\
(n=34)\end{array}$ & $\begin{array}{c}\text { Mujeres } \\
(n=38)\end{array}$ \\
\hline Edad & $20,1 \pm 2,9$ & $19,2 \pm 2,3$ & $28,1 \pm 6,1^{*}$ & $26,9 \pm 4,9 *$ \\
\hline Talla & $172,4 \pm 6,8$ & $158,7 \pm 5,9$ & $174,9 \pm 5,2^{*}$ & $160,2 \pm 5,0$ \\
\hline Peso & $66,2 \pm 11,7$ & $57,1 \pm 10,1$ & $76,1 \pm 12,6^{*}$ & $61,6 \pm 10,1^{*}$ \\
\hline
\end{tabular}

Datos mostrados como media \pm Desviación Estándar. Edad: años. Talla: cm. Peso: kg.

$*$ Diferencias significativas $\mathrm{p}<0,05$ con el grupo IC del mismo sexo.

Se tuvo como criterio general de inclusión no presentar impedimento físico para la realización de las pruebas; todos los sujetos fueron informados sobre el propósito y los riesgos del estudio y aprobaron su participación mediante la firma de un consentimiento informado. Se garantizó la protección de los datos y todos los procedimientos empleados se ajustaron a lo dispuesto en la Declaración de Helsinki (2008).

\section{Diseño}

Se realizó un estudio descriptivo de carácter exploratorio y corte transversal, en el cual se registró información sociodemográfica y del estado de salud, algunos parámetros antropométricos y pruebas de condición física en universitarios de ambos sexos, que a su vez conformaban los grupos IC y FC. Se hicieron comparaciones intergrupos por sexo.

\section{Procedimiento}

Se tomó información sociodemográfica (edad, sexo, semestre cursado), antecedentes familiares y personales de enfermedad, así como práctica deportiva actual, mediante una encuesta de respuestas cerradas elaborada específicamente para el estudio. En segunda instancia se evaluaron los componentes de la CFRS definidos por el Colegio Americano de Medicina del Deporte (ACSM): peso y composición corporal, capacidad aeróbica, fuerza muscular y flexibilidad (Garber et ál., 2011).

La talla se tomó con un tallímetro SECA Messband 206, el peso con una báscula de bioimpedancia eléctrica TANITA $®$ modelo BC-554 con 100 gramos de precisión. Se calculó el índice de masa corporal (IMC) dividiendo el peso sobre la talla expresada en metros al cuadrado. Para la medición de estas variables se tuvieron en cuenta las recomendaciones técnicas establecidas por la Sociedad Internacional para el Avance de la Cineantropometría - ISAK. La adiposidad se estimó mediante pesaje en la balanza de bioimpedancia eléctrica antes referenciada, teniendo en cuenta las indicaciones técnicas propias para este tipo de mediciones (Norton y Olds, 2000).

La flexibilidad isquiosural se evaluó mediante el Sit and Reach Test Modificado (SRTM) utilizando un flexómetro de madera ajustable a la altura de los pies y a la longitud de los miembros superiores (López-Miñarro, Alacid, Muyor y López, 2010). La prueba se hizo con el evaluado en posición sentado apoyando los hombros, la espalda y las espinas ilíacas en una pared lisa, mientras las piernas estaban extendidas apoyando los pies descalzos contra el flexómetro. Se aisló el efecto generado por la contribución de los músculos serratos a través de la protracción de las escápulas en el desplazamiento anterior de los miembros superiores, cuantificando este valor con el ejecutante en posición sentado y el resultado se restó del valor obtenido en la flexión profunda del tronco. Se asumió el mejor de dos intentos.

La fuerza muscular se evaluó en una de sus manifestaciones: la potencia de miembros inferiores. La evaluación se hizo mediante los saltos Abalakov y en contramovimiento (CMJ), empleando una plataforma de contactos Axon Jump Modelo T, un computador Dell Inspiron ${ }^{\circledR}$ y un software para la captura de la información provisto por el fabricante de la plataforma. Para cada técnica, el evaluado hizo tres intentos con una pausa de 2 minutos entre cada uno, partiendo de una flexión de rodillas aproximada a $90^{\circ}$ y se registró el mejor valor.

La aptitud cardiorrespiratoria ( $\mathrm{VO}_{2}$ máx.) se evaluó con el test de Rockport (caminata de la milla). Esta prueba y su ecuación predictiva originalmente fueron validadas por Kline et ál. (1987) para poblaciones entre 30 y 69 años, pero estudios posteriores viabilizaron la prueba para ser aplicada en poblaciones homólogas a la nuestra (Byars et ál., 2003; Dolgener, Hensley, Marsh y Fjelstul, 
1994) y convalidan la ecuación predictiva en edades menores (McSwegin, Plowman, Wolff y Guttenberg, 1998). Además se optó por utilizar esta prueba y su ecuación, porque la población a evaluar era heterogénea y el protocolo se consideró incluyente, adherente y menos riesgoso. La prueba consistió en recorrer 1609 metros caminando al mejor ritmo posible del ejecutante. Al final de la misma se registró el tiempo empleado con cronómetros Casio® HS-3, la frecuencia cardiaca con monitores de ritmo cardíaco Polar® y sensores T-31. Se empleó la ecuación predictiva de Kline et ál. (1987) para estimar el vo ${ }_{2}$ máx.:

$\mathrm{VO}_{2}$ máx. $=132.853-(0.0769 * \mathrm{P})-(0.3877 * \mathrm{E})+(6.315 * \mathrm{G})-(3.2649 * \mathrm{~T})-(0.1565 * \mathrm{FC})$

Donde:

\begin{tabular}{l|l|l|l|l} 
P: peso en libras & E : edad en años & $\begin{array}{c}\text { G : género (Hombres: 1 } \\
\text { y Mujeres: 0). }\end{array}$ & $\begin{array}{c}\text { T: tiempo para recorrer } \\
\text { la milla }\end{array}$ & $\begin{array}{c}\text { FC: frecuencia cardiaca } \\
\text { final }\end{array}$
\end{tabular}

Las evaluaciones, según correspondieran, se llevaron a cabo en el Laboratorio de Fisiología del Ejercicio del Área Educación Física y Deporte y el Centro Deportivo Universitario de la Universidad del Valle, Cali (Colombia). Todas las pruebas motoras realizadas contaron con ensayos previos y estímulos verbales por parte de los evaluadores, procurando el máximo esfuerzo del ejecutante.

\section{Análisis de datos}

El análisis de la información se hizo segmentado por sexo y grupo (IC-FC), se verificó la normalidad en la distribución de los datos con la prueba Kolmogorov-Smirnov y se hizo estadística descriptiva con distribuciones de frecuencias, media y desviación estándar. Las comparaciones entre grupos se hicieron con la Prueba $T$ para diferencia de medias en muestras independientes y la prueba de homogeneidad de varianzas de Levene. Todos los análisis se efectuaron con nivel de significan-

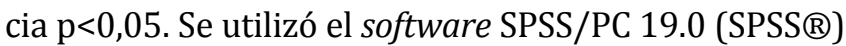
y la hoja electrónica Excel 2007 (Microsoft Corporation).

\section{RESULTADOS}

En la tabla 1 (ver sección Métodos) se señalaron diferencias entre la edad de los subgrupos, que muestran una media significativamente mayor en los grupos FC de hombres y mujeres. También se observan mayores valores en la talla de los hombres FC y en el peso para ambos sexos en el mismo grupo. Sobre los antecedentes familiares y personales de ECNT se observó similitud en los registros de los dos subgrupos estudiados, por lo que la información se presenta en uno solo. Así, el $38,3 \%$ de la población reportó que en su primer grado de consanguinidad hay presente al menos una de estas enfermedades. El mismo dato por sexo sitúa este factor de riesgo en el $41,6 \%$ de las mujeres y el $34,5 \%$ de los hombres que reportaron este antecedente familiar. La frecuencia relativa de las enfermedades que afectan al núcleo familiar se representa en la gráfica 1.
En relación con el propio estado de salud, el 96,4\% de la muestra estudiada se autorreconoció como sana. El 3,6\% restante se dividió en afecciones como cáncer, enfermedad coronaria, diabetes, hipertensión y dislipidemia. Por otra parte, la frecuencia de práctica deportiva se estableció mediante la existencia de al menos otro espacio formal de actividad adicional a la sesión de clase a la que concurrían los sujetos; los resultados de esta variable se presentan en la tabla 2.

Allí (tabla 2) puede observarse que en los grupos IC y FC el porcentaje de mujeres que realizaban práctica deportiva adicional a la sesión de clase es inferior al de los hombres, quienes incluso en el grupo IC las duplican. Llama la atención los porcentajes ligeramente superiores de las mujeres FC frente a las IC. Por su parte, los porcentajes de práctica adicional obtenidos en los hombres son bastante altos, incluso para los del grupo FC. En otro orden de ideas, los valores de los indicadores de CFRS en cada uno de los grupos se presentan en las tablas 3 y 4 .

En dichas tablas ( 3 y 4 ), se presentaron diferencias significativas en las mujeres, excepto para el SRTM y el CMJ (peso e IMC: $p<0,05$; adiposidad y salto Abalakov: $p<0,01 ; \mathrm{vo}_{2}$ máx. de $\left.p<0,001\right)$. En todos lo casos las diferencias siempre mostraron más valores desfavorables para las mujeres del grupo FC. En los hombres, todos los parámetros morfológicos y el $\mathrm{VO}_{2}$ máx. presentaron diferencias significativas $(p<0,001)$ y las dos pruebas de salto tuvieron diferencias significativas $p<0,05$. El SRTM fue la única variable que no tuvo diferencias. Similar a lo descrito para las mujeres, también hubo registros más desfavorables en el grupo de hombres FC. 
Gráfica 1. Antecedentes familiares de enfermedad crónica no transmisible

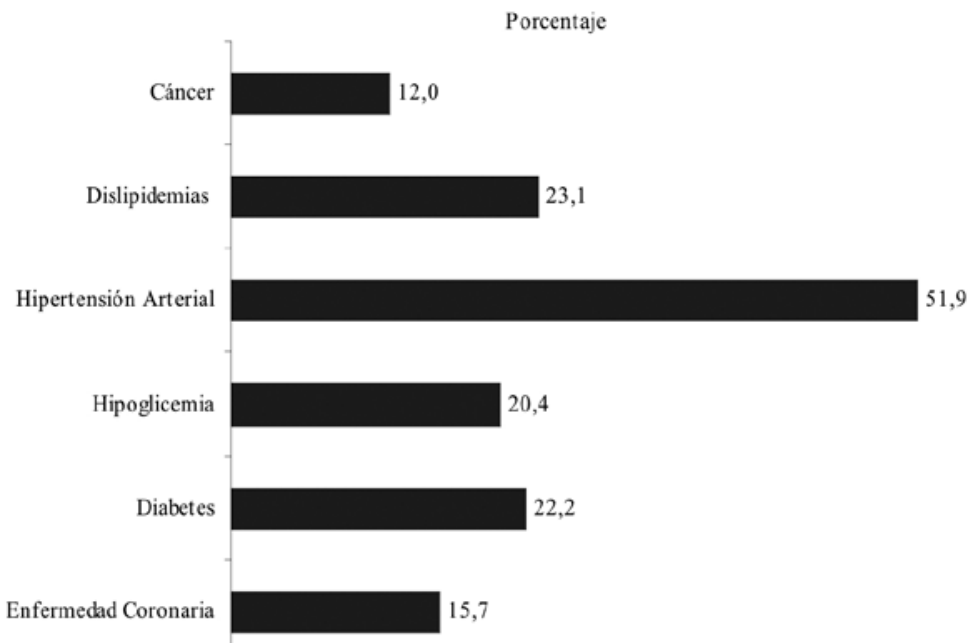

Tabla 2. Práctica deportiva adicional a la sesión de clase

\begin{tabular}{|c|c|c|c|c|}
\hline \multirow{2}{*}{$\begin{array}{c}\text { Práctica } \\
\text { deportiva }\end{array}$} & IC & Fu & \multicolumn{2}{c|}{ Hombres } \\
\cline { 2 - 5 } & 31,5 & 36,8 & 67,7 & FC \\
\hline Sí & 68,5 & 63,2 & 32,3 & 44,9 \\
\hline No & & & & 4 \\
\hline
\end{tabular}

IC: inicio de carrera. FC: fin de carrera.

Tabla 3. Valores de peso, IMc y adiposidad en los grupos del estudio

\begin{tabular}{|c|c|c|c|c|}
\hline & \multicolumn{2}{|c|}{ Mujeres } & \multicolumn{2}{|c|}{ Hombres } \\
\hline & $\begin{array}{c}\text { IC } \\
(n=111)\end{array}$ & $\begin{array}{c}\text { FC } \\
(n=38)\end{array}$ & $\begin{array}{c}\text { IC } \\
(n=97)\end{array}$ & $\begin{array}{c}\text { FC } \\
(n=34)\end{array}$ \\
\hline Peso & $57,1 \pm 10,1$ & $61,6 \pm 10,1^{*}$ & $66,2 \pm 11,7$ & $76,1 \pm 12,6^{*}$ \\
\hline IMC & $22,6 \pm 3,6$ & $24,1 \pm 4,4^{*}$ & $22,2 \pm 3,3$ & $24,9 \pm 4,3^{*}$ \\
\hline Adiposidad & $24,2 \pm 7,5$ & $28,9 \pm 6,9^{*}$ & $14,3 \pm 5,5$ & $19,4 \pm 6,8^{*}$ \\
\hline
\end{tabular}

Datos mostrados como media \pm desviación estándar. Peso: $\mathrm{kg}$. IMC: $\mathrm{kg} / \mathrm{m}^{2}$. Adiposidad: \%.

* Diferencias significativas $\mathrm{p}<0,05$ con el grupo IC del mismo sexo.

Tabla 4. Valores de condición física relacionada con la salud en los grupos del estudio

\begin{tabular}{|c|c|c|c|c|}
\hline & \multicolumn{2}{|c|}{ Mujeres } & \multicolumn{2}{|c|}{ Hombres } \\
\hline & $\begin{array}{c}\text { IC } \\
(n=111)\end{array}$ & $\begin{array}{c}\text { FC } \\
(n=38)\end{array}$ & $\begin{array}{c}\text { IC } \\
(n=97)\end{array}$ & $\begin{array}{c}\text { FC } \\
(n=34)\end{array}$ \\
\hline SRTM & $31,3 \pm 6,6$ & $30,6 \pm 7,3$ & $31,6 \pm 7,8$ & $30,4 \pm 6,7$ \\
\hline Abalakov & $25,9 \pm 4,7$ & $23,7 \pm 4,1^{*}$ & $40,3 \pm 5,4$ & $38,0 \pm 6,6^{*}$ \\
\hline CMJ & $22,3 \pm 3,9$ & $21,4 \pm 4,1$ & $34,7 \pm 4,8$ & $32,4 \pm 6,2^{*}$ \\
\hline vo 2 máx & $41,6 \pm 4,7$ & $36,4 \pm 5,9^{*}$ & $51,0 \pm 5$ & $44,8 \pm 5,6^{*}$ \\
\hline
\end{tabular}

Datos mostrados como media \pm desviación estándar. SRTM, Abalakov, CMJ: $\mathrm{cm} \mathrm{vO}_{2} \mathrm{máx}$ : ml*kg/min.

* Diferencias significativas $\mathrm{p}<0,05$ con el grupo IC del mismo sexo. 


\section{DISCUSIÓN}

Este estudio se propuso determinar y comparar algunos parámetros de CFRS en dos grupos de universitarios que diferían en el tiempo de permanencia en sus estudios (IC y FC). Este en primera instancia se manifestó en las diferencias intergrupo encontradas en la edad en ambos sexos, que, además de la incidencia que pudo tener en las comparaciones de los parámetros evaluados, podría interpretarse en el grupo FC como un indicador indirecto de los influjos que la vida universitaria podría tener sobre la CFRS en los estudiantes. No obstante, faltan estudios longitudinales que describan la dinámica de esta variable y en general su asociación con los factores de riesgo en universitarios a lo largo de su formación.

En cuanto a la historia familiar y personal de enfermedad, el 38,3\% del grupo estudiado refirió tener antecedentes familiares de ECNT, y la hipertensión arterial (HTA) fue la enfermedad más reportada (51,9\% de quienes refirieron antecedentes). Tales datos se asemejan a lo observado por Burke et ál. (1991) en un estudio donde se asociaron factores de riesgo e historia familiar de enfermedad en 5115 adultos estadounidenses de ambos sexos, blancos y negros, entre 18 y 30 años de edad: la hipertensión se reportó como antecedente en el $56 \%$ de los negros y el $44 \%$ de los blancos. Similar a nuestros datos, en este estudio fueron menores los antecedentes familiares para diabetes y enfermedad coronaria, entre otros. Por otra parte, en nuestro caso el propio estado de salud no parece ser un problema, pues solo el 3,4\% de los evaluados se declaró afectado por alguna enfermedad.

Con relación a la cantidad de práctica deportiva, si bien los sujetos del estudio participaban de una sesión semanal, esta frecuencia no cumpliría con las recomendaciones mínimas de actividad física para la salud sugeridas por el ACSM (Garber et ál., 2011), por lo que podrían asumirse como poco activos. No obstante, el porcentaje de mujeres de ambos grupos (IC y FC) que podrían calificarse de este modo está por debajo de los valores descritos para población adulta colombiana (Ministerio de la Protección Social, 2007), e incluso al compararse con los reportados para universitarios donde se ha identificado una alta prevalencia del sedentarismo (Astudillo \& Rojas, 2006; Martínez-Lemos, 2009). En el caso de los hombres de ambos grupos la situación es bastante diferente, pues se presumen bastante activos, lo cual no se relaciona con los reportes antes citados y podría ser una cualidad propia de la muestra.
En relación con lo hallado en las distintas variables de la CFRS, en la proporcionalidad y adiposidad corporal se encontraron valores para el IMC ubicados dentro de rangos de normalidad para los subgrupos IC de ambos sexos (mujeres $22,6 \pm 3,6$ y hombres $22,2 \pm 3$,3). El peso corporal tuvo un valor mayor en los grupos FC, lo que afecta el IMC para ambos sexos, ya que al compararse con los grupos IC fueron significativamente mayores $\mathrm{y}$ se ubicaron en el límite superior del rango de normalidad. Esto se reflejó en el hecho de que en el grupo Fc el sobrepeso afecta al 34,2\% de las mujeres y al 35,3\% de los hombres y la obesidad al 5,3 y al 11,8\% respectivamente. Los indicadores de sobrepeso para ambos sexos están ligeramente por encima de la tendencia nacional del $32,2 \%$ y en el caso de los hombres el $13,7 \%$ es más alto al compararse con los índices de obesidad (Ministerio de la Protección Social, 2007).

En cuanto a la adiposidad, según el ACSM (2010), indistintamente de la metodología usada, hay consenso en opiniones respecto a qué valores para el porcentaje de grasa del 10 al 22\% para hombres y del 20 al 32\% para mujeres se consideran saludables. Al comparar nuestros valores de porcentaje de grasa con los datos del Instituto Cooper de Dallas citados en la misma publicación (ACSM, $2010)$, las mujeres IC $(24,2 \pm 7,0)$ se ubican entre los percentiles $30-40$ y las FC $(28,9 \pm 6,9)$ entre $10-20$, que califican por debajo y muy por debajo de la mediana respectivamente; las puntuaciones bajas se entienden como inadecuadas. Para los hombres IC sus valores $(14,3 \pm 5,5)$ califican en el percentil 60 (P60) y los FC $(19,4 \pm 6,8)$ en el P30, indicadores que estarían alrededor de la mediana y por debajo de esta, respectivamente.

Lo mismo se observó al valorar la adiposidad frente a referentes específicos por edad y sexo para la bioimpedancia eléctrica, según la escala propuesta por Kyle, Genton, Slosman y Pichard (2001), donde los grupos IC se ubican entre el P25 y el P50 (segundo cuartil), y los FC entre el P50 y el P75 (tercer cuartil), es decir, están en el $50 \%$ central de la distribución. Contrario al caso de la tabla del Instituto Cooper de Dallas, las puntuaciones altas son consideradas como inconvenientes.

No obstante lo anterior, al comparar nuestros datos con las normativas establecidas en el estudio de Kyle et ál. (2001) y por el ACSM (2010), debe considerarse que estas se hicieron con base en datos obtenidos de población caucásica y los criterios normales de adiposidad pueden diferir en su significado al valorar colombianos, dado que nuestro trabajo incluyó población predominantemente mestiza, algunos afrodescendientes y 
amerindios. No se hallaron valores de referencia construidos en el país utilizando esta técnica y en la población estudiada. Lo comentado sobre la valoración del IMC y la adiposidad con las escalas citadas y las diferencias significativas entre los valores para porcentaje de grasa entre los grupos IC y FC revela que tanto la proporcionalidad como la masa grasa parecen acercarse a valores poco saludables en el grupo FC.

Sobre las pruebas motoras que evalúan la CFRs, los valores de flexibilidad isquiosural no difirieron significativamente entre sexos ni al compararlos entre los grupos IC y FC. Al confrontar nuestros registros con otros estudios, fueron menores a los hallados por López-Mínarro et ál. (2010) en universitarios españoles, y a los de mujeres estadounidenses del estudio de Minkler y Patterson (1994); los hombres de este mismo estudio tuvieron registros similares a los nuestros. La flexibilidad isquiosural es un parámetro que refleja la retracción de la musculatura extensora de la columna y flexora de los miembros inferiores, a su vez predictor de riesgo osteomuscular en estas zonas (Minkler y Patterson, 1994; López-Mínarro et ál., 2010); dicho riesgo se ha visto reflejado en la prevalencia del dolor de espalda en el 28,5\% de adultos colombianos entre 18 y 69 años, aunque dicha estimación aumenta en diez de los departamentos, donde los porcentajes fueron entre el $34 \mathrm{y}$ el 39\% (Ministerio de la Protección Social, 2007).

En relación con la potencia de miembros inferiores, un aspecto observado en los resultados fue que los promedios para el CMJ son menores y más desfavorables para ambos géneros en el grupo FC. Si bien este grupo es mayor en edad que el Ic, su promedio todavía no se sitúa en el rango en que esta cualidad normalmente empieza a decrecer, por lo cual consideramos este resultado como desfavorable frente a la prevención de enfermedades. Esto porque la potencia de miembros inferiores, más allá de reflejar la eficiencia de una determinada vía energética relacionada con una respuesta mecánica, expresa un estado de adaptación neuromuscular que puede asociarse con la funcionalidad de este sistema, por ser indicador de la capacidad de reclutamiento de unidades motoras rápidas y reflejo del estado funcional del tejido muscular (Moliner-Urdiales et ál., 2010b; Garber et ál., 2011).

Es importante anotar que la evaluación de esta manifestación de la fuerza por medio de saltos verticales $\mathrm{u}$ horizontales ha sido incluida en muchas de las más importantes baterías de pruebas de aptitud física en el mundo y es relativamente reciente su asociación con indicadores de salud, tema en el cual su mayor reconocimiento se ha hecho en la influencia sobre la capacidad funcional de personas mayores (Garber et ál., 2011). En el caso de población adolescente en el Estudio Helena se demostró que el acondicionamiento muscular, la velocidad y la agilidad estaban fuertemente relacionados con el estado de salud actual y futuro (Ortega et ál., 2011b).

No obstante, la bibliografía sigue siendo contradictoria en este aspecto para el caso de los adolescentes, pues en los hombres del Estudio Helena se describieron asociaciones positivas entre la actividad física vigorosa y las pruebas de fuerza en miembros inferiores, aunque esto no calificó para el CMJ; todo lo anterior no se observó en las mujeres (Moliner-Urdiales et ál., 2010b). En general son escasos los parámetros que relacionen la potencia con la salud en jóvenes universitarios de distinto nivel de condición física y, en nuestro caso particular, de características étnicas diversas, por lo que nuestros registros no fueron susceptibles de ser comparados. La generación de un perfil para la potencia de miembros inferiores con escalas de valoración relacionadas con la salud y la funcionalidad en población homóloga a la nuestra es un pendiente a desarrollar en Latinoamérica.

Sobre la resistencia cardiorrespiratoria, para la comparación de nuestros valores de $\mathrm{vo}_{2}$ máx. se tomó como población de referencia la representada en los datos del Instituto Cooper de Dallas citados por el ACSM (2010). Lo compilado en los textos de esta organización, en su época, también fue referente de valoración en el estudio de Dolgener et ál. (1994), quienes en la predicción del $\mathrm{vo}_{2}$ máx. relativo, con la ecuación de Kline et ál. (1987), hallaron promedios de 46,3 $\pm 8,01 \mathrm{ml} * \mathrm{~kg} / \mathrm{min}$ en hombres y de $36,3 \pm 4,73 \mathrm{ml} * \mathrm{~kg} / \mathrm{min}$ en mujeres, valores que los autores calificaron como característicos de estudiantes universitarios. Byars et al. (2003) usando la misma ecuación en estudiantes homólogos, hallaron promedios menores ( $40.66 \pm 4.98$ en hombres; 34.45 \pm 4.48 en mujeres).

En comparación con estos datos, nuestros valores fueron mayores y al situarlos frente a los referentes del Instituto Cooper (ACSM, 2010), las mujeres y los hombres al inicio de su vida universitaria se ubicaron por encima de la mediana poblacional (P70 y entre el P70 y el P80 respectivamente), asunto que en cierto modo contradice la evidencia sobre el bajo nivel de acondicionamiento relacionado con la salud en los adolescentes que se ha señalado como tendencia mundial (Moliner-Urdiales et ál. 2010a; Ortega et ál., 2011b) y que sería esperable en los jóvenes que ingresan a la educación superior. 
No obstante, en un reporte de Moliner-Urdiales et ál. (2010a) se refirió una mejoría en los niveles de velocidad, agilidad y resistencia en adolescentes de Zaragoza (España) seguidos longitudinalmente y que habían tomado parte de los estudios Avena y Helena. En el mismo sentido, Ortega et ál. (2011b) reportaron que el $61 \%$ de los hombres y el $57 \%$ de las mujeres adolescentes europeos del Estudio Helena tienen niveles de acondicionamiento cardiorrespiratorio saludable, mostrando una mejoría de esta condición en comparación con estudios de décadas anteriores. Esto contradice las tendencias seculares de disminución en el acondicionamiento de este grupo etario que ha descrito la literatura. Dada la solidez metodológica de ese estudio, las limitaciones de comparación con otros por la variedad de métodos usados en la estimación de la aptitud física y los pocos reportes con diseños longitudinales, los autores sugirieron una probable detención en la caída de estos indicadores y lo justificaron en un posible efecto de las iniciativas institucionales de salud pública, orientadas a mejorar condiciones nutricionales y niveles de actividad física, que fueron emprendidas en España y Europa desde 2005.

Es difícil acoger esta misma explicación para el caso del buen nivel de aptitud cardiorrespiratoria observado en nuestros estudiantes del grupo más joven, pues no se ha documentado algo similar en Colombia, ni sobre relaciones entre resultados de las estrategias de promoción de estilos de vida activos y la práctica deportiva con fines de salud, con los efectos sobre la CFRs de nuestros jóvenes; por lo que otro aspecto a revisar tiene que ver con el método de estimación del $\mathrm{vo}_{2}$ máx. utilizado en este estudio, respecto del cual Dolgener et ál. (1994) habían señalado una posible sobreestimación en los sujetos de baja forma física. Estos autores dicen que por las características del nivel de acondicionamiento de los sujetos que conformaron la muestra del estudio de Kline et ál. (1987), sobre todo los del rango etario de 30-39 años, la ecuación sobreestima el $\mathrm{vo}_{2}$ máx. de los evaluados con bajo nivel de forma física y subestima los de alto nivel.

Nuestros valores obtenidos con esta ecuación son altos, aunque nuestra población fue heterogénea en su capacidad física, por lo tanto, generalizar una conclusión respecto al hecho que el $\mathrm{vo}_{2}$ máx. pueda estar sobreestimado o subestimado en nuestro caso no es pertinente. Además, en el análisis exploratorio de los datos se estimó el $\mathrm{VO}_{2}$ máx. con la ecuación de Dolgener et ál. (1994) y los resultados fueron valores más altos, cuando supuestamente el modelo predictivo propuesto por estos autores corregía la sobreestimación declarada para el de Kline et ál. (1987). En conclusión, los altos valores de $\mathrm{vo}_{2}$ máx. estimados en los hombres pueden explicarse en que un porcentaje superior al $60 \%$ refirió tener una práctica deportiva constante, lo cual seguramente influyó en esos registros. Esta razón no puede atribuirse al caso de los valores obtenidos en las mujeres.

En el caso de los subgrupos FC, al comparar con la población de referencia (ACSM, 2010), el promedio para el $\mathrm{VO}_{2}$ máx. de los hombres se situó en la media (entre P50 y P60) y el de las mujeres por debajo de esta (P40), valores que fueron menores y significativos al compararse con los promedios de quienes iniciaban los estudios.

Todos estos comportamientos pueden atribuirse a las diferencias etarias entre los grupos. De este modo, según los promedios de edad, los dos subgrupos (IC y FC) se ubican al inicio de la segunda década de vida y en la segunda mitad de esta respectivamente, periodo en el cual se esperaría una estabilidad en la manifestación de las capacidades condicionales, concretamente el $\mathrm{VO}_{2}$ máx.; al no observarse, es posible especular sobre la influencia que, además de la edad, podrían tener los influjos de la vida universitaria y particularmente aquellos que condicionen el nivel de aptitud física de los estudiantes y que afecten la dinámica de esta capacidad condicional. Sin embargo el diseño metodológico de este estudio no puede aclararlo suficientemente.

Con lo anterior, en general hay un aspecto en el cual nuestros resultados no son consistentes con lo reportado en otras fuentes bibliográficas; es el relacionado con el bajo nivel de actividad física que se ha referido y que resultaría característico de poblaciones universitarias de Iberoamérica (Astudillo y Rojas, 2006; Castillo y Giménez, 2011; Martínez-Lemos, 2009; Martins et ál., 2008; Oviedo et ál., 2008). Nuestros resultados reflejan que la aptitud física de la población estudiada está en niveles aceptables, muy a pesar de que las mujeres declararon menor frecuencia de realización de actividades deportivas. Una explicación de esta falta de coherencia tiene que ver con la diversidad de metodologías empleadas, que difícilmente permiten comparar objetivamente los hallazgos. Adicionalmente, en muchos de los estudios que valoran riesgo en universitarios se indaga por la actividad física de los sujetos, muchas veces por medio de encuestas; este concepto es supremamente ambiguo por la generalidad que implica.

Nuestra metodología incluyó una prueba aeróbica que permitiera estimar la aptitud física relacionada con la 
salud y que por las condiciones de validez y objetividad de los registros, así como por la importancia que este componente ha cobrado en los estudios de predicción de riesgo cardiovascular (Ortega et ál., 2008), se considera útil hacer aproximaciones desde la evaluación de las capacidades condicionales, más que desde estimaciones de gasto energético, toda vez que la baja aptitud física, de forma excluyente, en sí misma es un factor de riesgo que explica un gran porcentaje de la morbimortalidad (García, et ál., 2007; Pagac, 2008).

\section{CONCLUSIÓN}

Si bien la población estudiada se autorreconoce como sana, la CFRS difiere entre los grupos IC y FC en ambos sexos, mostrando valores más desfavorables para todos los parámetros en el grupo $\mathrm{FC}$, lo que podría ser atribuible a su mayor edad. No obstante, el $\mathrm{Vo}_{2}$ máx. en ambos grupos denota una aceptable CFRs, lo que contradice datos sobre prevalencia de sedentarismo y baja forma física previamente informados en universitarios. Futuros estudios podrían indagar la dinámica de la CFRS a lo largo de este ciclo educativo

\section{AGRADECIMIENTOS}

El autor agradece al médico Álex Estrada Juri y su empresa Polar-Cali, por el préstamo de monitores de frecuencia cardiaca. También al colega Francisco Antonio Amú por el apoyo en el procesamiento de la información y al equipo de trabajo del Grupo de Investigación Incide por la colaboración en las evaluaciones hechas para este estudio.

\section{REFERENCIAS BIBLIOGRÁFICAS}

American College of Sports Medicine -ACSM. (2010). Guidelines for exercise testing and prescription (pp. 66-80). Seventh Edition. Philadelphia; Lippincon Williams y Wilkins: $66-80$.

Ardoy, D., Fernández, J., Chillón, P., Artero, E., España, V., Jiménez, D., Ruiz, J., Guirado, C., Castillo, M. y Ortega, F. B. (2010). Educando para mejorar el estado de forma física, Estudio Edufit: antecedentes, diseño, metodología y análisis del abandono/adhesión al estudio. Revista Española de Salud Pública, 84(2), 151-168.

Astudillo-García, C., Rojas-Russell M. (2006). Autoeficacia y disposición al cambio para la realización de actividad física en estudiantes universitarios. Bogotá. Acta Colombiana de Psicología, 9(1), 41-49.

Burke, G., Savage, P., Sprafka, J., Selby, J., Jacobs, J., Perkins, L., et al. (1991). Relation of risk factor levels in young adulthood to parental history of disease. The CARDIA study. Circulation, 84(3), 1176-1187.

Byars, A., Greenwood, M., Greenwood, L. y Simpson, W. (2003). The effect of alternating steady-state walking technique on estimated $\mathrm{vo}_{2}$ max values of the Rockport Fitness Walking Test in college students. Official Journal of the American Society of Exercise Physiologist, 6, 21-25.

Castillo, E. y Giménez, F. (2011). Hábitos de práctica de actividad física del alumnado de la Universidad de Huelva. Revista Internacional de Medicina y Ciencias de la Actividad Física y el Deporte, 10(41), 127-144.

Declaración de Helsinki. (2008). Principios éticos para las investigaciones médicas en seres humanos. Asociación Médica Mundial.

Dolgener, F., Hensley, L., Marsh, J. y Fjelstul, J. (1994). Validation of the Rockport Fitness Walking Test in College Males and Females. Research Quarterly for Exercise and Sport, 65(2), 152-158.

Garber, C. E., Blissmer, B., Deschenes, M.R., Franklin, B.A., Lamonte, M.J., Lee, I.M., Nieman, D.C. y Swain, D. P. (2011). American College of Sports Medicine position stand. Quantity and quality of exercise for developing and maintaining cardiorespiratory, musculoskeletal, and neuromotor fitness in apparently healthy adults: guidance for prescribing exercise. Medicine and Science in Sports and Exercise, 43(7), 1334-1359.

García, E., Ortega, F., Ruiz, J., Mesa, J., Delgado, M., González-Gross, M., García-Fuentes, M., Vicente, G., Gutiérrez, A. y Castillo, J. M. (2007). El perfil lipídico-metabólico en los adolescentes está más influido por la condición física que por la actividad física (Estudio Avena). Revista Española de Cardiología, 60(6), 581-588.

Kline, G.; Porcari, J.; Hintermeister, R.; Freedson, P.; Ward, A.; McCarron, R.; Ross, H. y Rippe, J., (1987). Estimation of $\mathrm{VO}_{2}$ máx. from a one-mile track walk, gender, age, and body weight. Medicine and Science in Sports and Exercise, 19(3), 253-259.

Kyle U., Genton, L, Slosman, D. y Pichard, C. (2001). Fatfree and fat mass percentiles in 5225 healthy subjects aged 15 to 98 years. Nutrition, 17(7), 534-541. 
López-Miñarro, P., Alacid, F., Muyor, J. y López, F. (2010). Validez del test sit-and-reach modificado como criterio de extensibilidad isquiosural en adultos jóvenes. Kronos, 9 (17), 39-46.

Martínez-Gómez, D., Eisenmann J. C., Gómez-Martínez, S., Veses, A., Marcos, A. y Veiga, O. (2010). Sedentarismo, adiposidad y factores de riesgo cardiovascular en adolescentes. Estudio Afinos. Revista Española de Cardiología, 63(3), 277-285.

Martínez-Gómez, D., Ortega, F., Ruiz, J., Vicente-Rodríguez, G., Veiga, O. L., Widhalm, K. y Sjöström, M. (2011). Excessive sedentary time and low cardiorespiratory fitness in European adolescents: the HELENA study. Archives of Disease in Childhood, 96(3): 240-246.

Martínez-Lemos, R. I. (2009). Prevalencia y factores asociados al hábito sedentario y a la intención de práctica de actividad física en alumnado universitario. Revista de Investigación en Educación, 6, 193-194.

Martins, F., De Castro, M., de Santana, G., y Oliveira, L. (2008), Estado nutricional, medidas antropométricas, nivel socioeconómico y actividad física en universitarios brasileños. Nutrición Hospitalaria, 23(3), 234-241.

McSwegin, P.; Plowman, S.; Wolff, G. y Guttenberg, G. L. (1998). The Validity of a One-Mile Walk Test for High School Age Individuals. Measurement in Physical Education and Exercise Science, 2(1), 47-63.

Mendes, J., García, T., Sánchez, P.A., Menayo, R., Cervelló, E. (2006). A Composição Corporal, o vo máx. e o estilo de vida de jovens estudantes do ensino superior. Revista de Desporto e Saúde da Fundação Técnica e Científica do Desporto, 4(4), 73-79.

Ministerio de la Protección Social, República de Colombia. (2007). Encuesta Nacional de Salud. Disponible en: http://www.minsalud.gov.co/Documentos $\% 20$ y\%20Publicaciones/Encuesta\%20Nacional.pdf.

Minkler, S. y Patterson, P. (1994). The validity of the modified sit-and-reach test in college-age students. Research Quarterly for Exercise and Sport, 65(2), 189-192.

Moliner-Urdiales, D., Ruiz, J., Ortega, F., Jiménez-Pavón, D., Vicente-Rodriguez, G., Rey-López, J., Martínez, D., Casajús, J., Mesana, M., Marcos, A., Noriega-Borge, M., Sjöström, M., Castillo, M., Moreno, L. (2010a). On behalf of the AVENA and HELENA Study Groups. Secular trends in health-related physical fitness in Spanish adolescents: The AVENA and HELENA Studies. Journal of Science and Medicine in Sport, 13(6), 584-588.

Moliner-Urdiales, D., Ortega, F., Vicente, G., Rey, J., Gracia, L., Kurt Widhalm, K., Sjöström, M., Moreno, L., Castillo, M. y Ruiz, J. (2010b). Association of physical activity with muscular strength and fat-free mass in adolescents: the HELENA study. European Journal of Applied Physiology, 109(6), 1119-1127.

Norton, K. y Olds, T. (2000). Antropométrica (pp. 157 184). Rosario: Biosystem.

Ortega, F., Artero, E., Ruiz, J., España-Romero, V., Jiménez, D., Vicente-Rodríguez, G., Moreno, L., Manios, Y., Béghin, L., Ottevaere, C., Ciarapica, D., Sarri, K., Dietrich, S., Blair, S., Kersting, M., Molnar, D., González-Gross, M., Gutiérrez, Á., Sjöström, M., y Castillo, M. (2011b). On behalf of the HELENA study group. Physical fitness levels among European adolescents: the HELENA study. British Journal of Sports Medicine, 45(1), 20-29.

Ortega, F., Ruiz, J., Hurtig-Wennlöf, A. y Sjöström, M., (2008). Los adolescentes físicamente activos presentan una mayor probabilidad de tener una capacidad cardiovascular saludable independientemente del grado de adiposidad. The European Youth Heart Study. Revista Española de Cardiología, 61(2), 123-129.

Oviedo, G., De Salim, A. M., Santos, I., Sequera, S., Soufrontt, G., Suárez, P., y Arpaia, A. (2008). Factores de riesgo de enfermedades crónicas no transmisibles en estudiantes de la carrera de Medicina. Universidad de Carabobo. Nutrición Hospitalaria, 23(3), 288-293.

Physical Activity Guidelines Advisory Committee. (Pagac). Physical Activity Guidelines Advisory Committee Report, 2008. Washington, DC: us Department of Health and Human Services, 2008, A1-H14.

Ramos, S., Alzate, D., Ayala, J., Franco, A. y Sánchez, J. (2009). Perfil de fitness de los estudiantes de la Universidad de Caldas. Colombia. Hacia la Promoción de la Salud, 14(1) 23-34.

Vizcaíno, F. M., Aguilar, F. S., Artalejo, F. R., Vizcaíno, V. M., Luz Domínguez Contreras, M., y Regidor, R. T. (2002). Prevalencia de la obesidad y mantenimiento del estado ponderal tras un seguimiento de 6 años en niños y adolescentes: estudio de Cuenca. Medicina Clínica, 119(9), 327-330. 